\title{
Short- and long-term follow-up of patients with non-neoplastic esophageal perforation
}

\author{
Sebastian Brinkmann ${ }^{1,2}$ (1) $\cdot$ Laura Knepper ${ }^{1} \cdot$ Hans Fuchs $^{1} \cdot$ Arnulf Hoelscher ${ }^{3} \cdot$ Kathrin Kuhr $^{4}$. \\ Daniel Pinto dos Santos ${ }^{5}$. Patrick Plum ${ }^{1}$. Seung-Hun Chon ${ }^{1}$. Christiane Bruns ${ }^{1}$. Wolfgang Schroeder ${ }^{1}$. \\ Jessica Leers ${ }^{1}$
}

Received: 29 June 2021 / Accepted: 4 September 2021 / Published online: 25 September 2021

(c) The Author(s) 2021

\begin{abstract}
Purpose Esophageal perforation is associated with high morbidity and mortality. In addition to surgical treatment, endoscopic endoluminal stent placement and endoscopic vacuum therapy (EVT) are established methods in the management of this emergency condition. Although health-related quality of life (HRQoL) is becoming a major issue in the evaluation of any therapeutic intervention, not much is known about HRQoL, particularly in the long-term follow-up of patients treated for non-neoplastic esophageal perforation with different treatment strategies. The aim of this study was to evaluate patients' outcome after non-neoplastic esophageal perforation with focus on HRQoL in the long-term follow-up.

Methods Patients treated for non-neoplastic esophageal perforation at the University Hospital Cologne from January 2003 to December 2014 were included. Primary outcome and management of esophageal perforation were documented. Longterm quality of life was assessed using the Gastrointestinal Quality of Life Index (GIQLI), the Health-Related Quality of Life Index (HRQL) for patients with gastroesophageal reflux disease (GERD), and the European Organization for Research and Treatment of Cancer (EORTC) questionnaires for general and esophageal specific QoL (QLQ-C30 and QLQ-OES18). Results Fifty-eight patients were included in the study. Based on primary treatment, patients were divided into an endoscopic $(n=27 ; 46.6 \%)$, surgical $(n=20 ; 34.5 \%)$, and a conservative group $(n=11 ; 19 \%)$. Short- and long-term outcome and quality of life were compared. HRQoL was measured after a median follow-up of 49 months. HRQoL was generally reduced in patients with non-neoplastic esophageal perforation. Endoscopically treated patients showed the highest GIQLI overall score and highest EORTC general health status, followed by the conservative and the surgical group.

Conclusion HRQoL in patients with non-neoplastic esophageal perforation is reduced even in the long-term follow-up. Temporary stent or EVT is effective and provides a good alternative to surgery, not only in the short-term but also in the long-term follow-up.
\end{abstract}

Keywords Non-neoplastic esophageal perforation $\cdot$ Health-related quality of life $\cdot$ Long-term follow-up $\cdot$ Endoscopic endoluminal stent placement $\cdot$ Endoscopic vacuum therapy $\cdot$ Transthoracic esophagectomy

Sebastian Brinkmann

sebastian.brinkmann@uk-koeln.de

1 Department of General, Visceral, Cancer and Transplantation Surgery, University of Cologne, Kerpener Str. 62, 50937 Cologne, Germany

2 Department of General, Visceral and Vascular Surgery, Marien Hospital Herne, Ruhr University Bochum, Hölkeskampring 40, 44625 Herne, Germany
3 Department of General, Visceral and Trauma Surgery, Elisabeth-Krankenhaus Essen, Essen, Germany

4 Institute of Medical Statistics, Informatics and Epidemiology, University of Cologne, Cologne, Germany

5 Department of Radiology, University of Cologne, Cologne, Germany 


\section{Background}

Despite recent progress in surgical and endoscopic therapy, non-neoplastic esophageal perforation is still associated with high morbidity and mortality [1]. Various surgical, endoscopic, and conservative treatment strategies are available [2, 3]. Important for the outcome and for treatment choice is the extent of wound cavity, time of diagnosis, clinical inflammatory response, localization, and cause of perforation. There is a high variety of causes of esophageal perforation including spontaneous perforation, namely Boerhaave syndrome, iatrogenic perforation due to routine diagnostic endoscopy or transesophageal ultrasound, and rare traumatic causes. This variety leads to wide heterogeneity of treatment modalities resulting in an individualized treatment strategy for each patient based on cause of perforation. Over the last decades, treatment of esophageal perforation has changed from surgical treatment with diversion to more interventional treatment with stenting or endoscopic vacuum treatment.

Several studies have reported promising results in the short-term follow-up after effective treatment of this devastating complication [4, 5]. However, data on long-term follow-up are rare and especially HRQoL remains unclear. This study reports early outcome and efficacy of current treatment modalities and investigates patients' outcome after non-neoplastic esophageal perforation with focus on HRQoL in the long-term followup. Furthermore, the study aims to analyze the association between long-term outcome and different treatment modalities.

\section{Methods}

\section{Patients and data collection}

Patients who were treated for non-neoplastic esophageal perforation at the University Hospital Cologne between January 2003 and December 2014 were included in this study. They were identified from a prospectively maintained database, and outcome data were recorded in a phone- and/or mail-based interview using the HealthRelated Quality of Life (HRQoL) questionnaires. At the onset of the study, 25 of 58 (43.1\%) patients were already deceased. Of the remaining 33 patients, a total of 20 were available for the survey.

Diagnosis was established mainly by CT imaging and endoscopy. Initial diagnostic procedure was predominantly based on etiology and site of esophageal perforation. Primarily established therapeutic management was based on the cause, site and size of perforation, and on overall health status of the patient and signs of sepsis. In sum, diagnostic and therapeutic management did not follow a standardized protocol and were individualized for each patient. Demographic and clinical data of patients were collected, including patient characteristics; time and date of diagnosis; cause, site, and length of esophageal perforation; and treatment specifics. Peri- and postoperative complications, length of ICU and hospital stay, and mortality were analyzed. The protocol of this study was approved by the Ethics Committee of the University Hospital of Cologne (reference number 16-268).

\section{Health-related quality of life assessment}

HRQoL was assessed using four questionnaires: the HealthRelated Quality of Life Index for GERD, the Gastrointestinal Quality of Life Index (GIQLI), and the EORTC QLQ-C30 and QLQ-OES18 [6-8].

HRQoL for GERD contains 11 items. Ten of the items ask for symptoms and are scored from 0 to 5 , with a higher score indicating more severe symptoms. One item asks for satisfaction with the patients' present condition which can be stated as satisfied, neutral, or unsatisfied.

The GIQLI measures quality of life in patients with gastrointestinal diseases. It includes 36 items with scores ranging from 0 to 4, resulting in an overall score between 0 and 144. Scores can also be divided into five domains: symptoms, emotional function, physical function, social function, and effect of medical treatment. Higher scoring represents a better HRQoL.

Additional HRQoL data were generated with the EORTC QLQ-C30 (version 3.0) questionnaire and the esophageal cancer specific module QLQ-OES18. The QLQ-C30 was developed to measure HRQoL in cancer patients and consists of 30 items. The items can be combined to assess five functional scales (physical, role, cognitive, emotional, social), nine symptom scales (fatigue, nausea/vomiting, pain, dyspnea, insomnia, appetite loss, constipation, diarrhea, financial difficulties), and one global health status scale. QLQ-OES18 includes nine symptom scales (eating, reflux, pain, trouble swallowing saliva, choked when swallowing, dry mouth, trouble with taste, trouble with coughing, trouble talking) and one functional scale (dysphagia). Answers are expressed on scales ranging from 0 to 100 according to the EORTC scoring manual [9]. Higher scores in functional and global health status scales indicate a high or healthy level of functioning, whereas higher symptom scales indicate a high level of symptoms. Missing data were handled according to the EORTC scoring manual. 


\section{Statistical analysis}

Data were entered in Excel (Microsoft Office 365, Microsoft Corporation, Redmond, USA). Statistical analysis was performed using SPSS Statistics 23 (IBM Corporation, Armonk, USA). Due to the number of patients, the Mann-Whitney $U$ test or Kruskal-Wallis test for all metric and Fisher's exact test for all categorical variables were used. A $p$-value $<0.05$ was considered statistically significant.

\section{Results}

\section{Patients}

Fifty-eight patients were included in the study. The study cohort consisted of 29 men and 29 women, with a mean age of 65 years at the time of diagnosis (range: 21-92). Most common cause of esophageal perforation was iatrogenic $(n=30,51.7 \%)$, followed by spontaneous $(n=16$,
$27.6 \%)$ and traumatic perforation $(n=5,8.6 \%)$. Further demographics and clinical details of patients are shown in Table 1. Based on primary treatment choice, patients were divided into three groups: endoscopic, surgical, and conservative.

With the upcoming endoscopic treatment options in the latest years, primary treatment selection shifted from surgical to mainly endoscopic therapy as shown in Fig. 1.

Twenty-seven patients (46.6\%) were treated endoscopically. Esophageal stenting was performed in 18 patients with a median duration of stent placement of 33 days (range: 14-82). Stents were changed once on average, twice at the most. Two patients were treated with endoscopic vacuum therapy (EVT) with a median sponge inlay of 10.5 days and at least two endoscopic changes. Seven patients received a combined treatment of stent and EVT with a median stent inlay of 9 days (range: 4-50) and a median EVT of 10 days (range: 4-27) and a median sponge changing of 2 (range: $1-7$ ).
Table 1 Demographic and clinical characteristics of 58 patients with esophageal perforation (non-follow-up and follow-up patients)

\begin{tabular}{|c|c|c|c|c|}
\hline Variables & $\begin{array}{l}\text { All }(n=58) \\
n(\%)\end{array}$ & $\begin{array}{l}\text { Non-follow-up }(n=38) \\
n(\%)\end{array}$ & $\begin{array}{l}\text { Follow-up }(n=20) \\
n(\%)\end{array}$ & $p$ value \\
\hline Gender, female & $29(50)$ & $18(47.4)$ & $11(55)$ & 0.783 \\
\hline Age (years) & $64.6(\mathrm{SD} 13.9)$ & $64.9(\mathrm{SD} 15.1)$ & $64(\mathrm{SD} 11.6)$ & 0.600 \\
\hline BMI $\left(\mathrm{kg} / \mathrm{m}^{2}\right)$ & $24.4(\mathrm{SD} 4.4)$ & $24.6(\mathrm{SD} 4.1)$ & $24.1(\mathrm{SD} 5.1)$ & 0.404 \\
\hline Smoking & $9(15.5)$ & $8(21.1)$ & $1(5)$ & 0.143 \\
\hline Alcohol & $11(19.0)$ & $11(28.9)$ & $0(0)$ & 0.011 \\
\hline Comorbidity & $55(94.8)$ & $36(94.7)$ & $19(95)$ & 1.000 \\
\hline Arterial hypertension & $27(46.6)$ & $18(47.4)$ & $9(45)$ & 1.000 \\
\hline Atrial fibrillation & $17(29.3)$ & $11(28.9)$ & $6(30)$ & 1.000 \\
\hline COPD & $10(17.2)$ & $7(18.4)$ & $3(15)$ & 1.000 \\
\hline Coronary heart disease & $10(17.2)$ & $9(23.7)$ & $1(5)$ & 0.141 \\
\hline Diabetes & $7(12.1)$ & $6(15.8)$ & $1(5)$ & 0.403 \\
\hline Gastric and duodenal ulcers & $6(10.3)$ & $3(7.9)$ & $3(15)$ & 0.405 \\
\hline Achalasia & $4(6.9)$ & $1(2.6)$ & $3(15)$ & 0.114 \\
\hline \multicolumn{5}{|l|}{ Cause of perforation } \\
\hline Iatrogen & $30(51.7)$ & $19(50)$ & $11(55)$ & 0.787 \\
\hline Spontaneous & $16(27.6)$ & $12(31.6)$ & $4(20)$ & 0.538 \\
\hline Traumatic & $5(8.6)$ & $2(5.3)$ & $3(15)$ & 0.328 \\
\hline Other & $4(6.9)$ & $3(7.9)$ & $1(5)$ & 1.000 \\
\hline Unknown & $3(5.2)$ & $2(5.3)$ & $1(5)$ & 1.000 \\
\hline \multicolumn{5}{|l|}{ Site of perforation } \\
\hline Proximal & $10(17.2)$ & $2(5.3)$ & $8(40)$ & 0.002 \\
\hline Middle & $21(36.2)$ & $14(36.8)$ & $7(35)$ & 1.000 \\
\hline Distal & $27(46.6)$ & $22(57.9)$ & $5(25)$ & 0.026 \\
\hline Size of perforation $(\mathrm{cm})$ & $2(0.4-11)$ & $2(0.4-11)$ & $2(1-6)$ & 0.627 \\
\hline Delayed diagnosis $>24 \mathrm{~h}$ & $20(34.5)$ & $11(28.9)$ & $9(45)$ & 0.362 \\
\hline Delayed diagnosis (days) & $4.5(2-35)$ & $9(2-35)$ & $3(2-7)$ & 0.142 \\
\hline
\end{tabular}

Categorical variables are expressed as number (percentage); continuous variables are expressed as mean (standard deviation) 
Fig. 1 Development of treatment selection for esophageal perforation over time

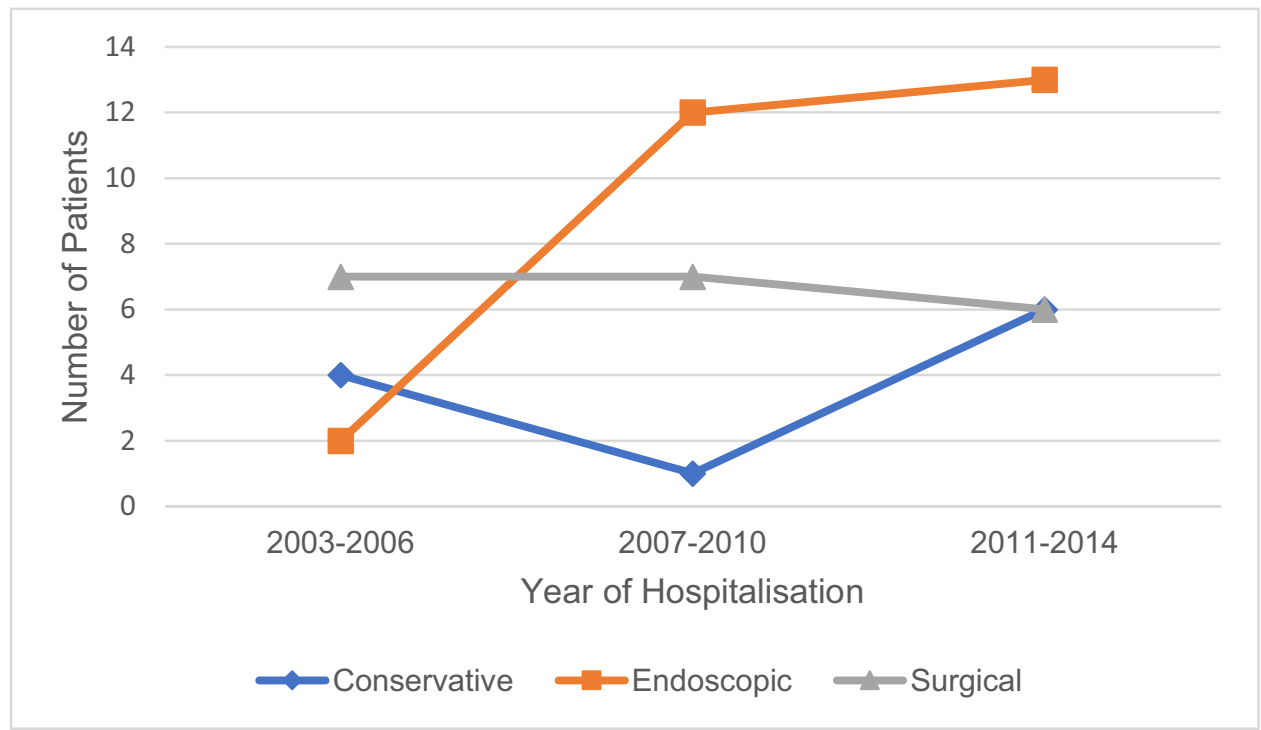

Surgery was performed in 20 patients $(34.5 \%)$. Fifteen patients initially received a transthoracic esophagectomy. Cervical diversion was required in 13 of these patients (65\%). In 7 patients, gastrointestinal reconstruction was performed later on. The remaining 5 patients were treated with primary suture of the perforated area. In three of these patients, transthoracic esophagectomy was performed in the follow-up $(2 \times$ cervical deviation, $1 \times$ intrathoracic esophagogastrostomy). Median time to reconstruction of cervical esophagostomy was 11.5 months (range: 6-42).

Conservative management was applied in 11 patients (19\%). Therapy mainly consisted of antibiotics, proton pump inhibitors, parenteral nutrition, and in 5 cases chest tube drainage.

\section{Outcome}

Outcome parameters were compared between treatment groups to identify possible differences. Overall, median hospital stay was 31.5 days (range 6-243), and median ICU stay was 9 days (range 1-200). Both were significantly longer in patients treated with surgery $(p=0.017 ; 0.003)$. Patients suffered from various complications including pleural effusion and empyema or pneumonia. Complication rate was highest in the surgical group with $85 \%$, compared to $72.7 \%(n=8)$ in the conservative and $81.5 \%(n=22)$ in the endoscopic group. Even though no statistical difference could be demonstrated, thirteen patients had to return to the hospital for further therapy of late complications like esophageal stenosis, most of whom had been treated endoscopically $(n=8,29.6 \%)$. Overall, 90-day mortality rate was $13.8 \%(n=8,4$ endoscopic, 2 conservative, 2 surgical). Further details are displayed in Table 2.

Table 2 Short-term outcome stratified by treatment groups

\begin{tabular}{|c|c|c|c|c|c|}
\hline Variables & $\begin{array}{l}\text { All }(n=58) \\
n(\%)\end{array}$ & $\begin{array}{l}\text { Conservative }(n=11) \\
n(\%)\end{array}$ & $\begin{array}{l}\text { Endoscopic }(n=27) \\
n(\%)\end{array}$ & $\begin{array}{l}\text { Surgical }(n=20) \\
n(\%)\end{array}$ & $p$ value \\
\hline Complications & $42(72.4)$ & 7 (63.6) & $18(66.7)$ & $17(85)$ & 0.279 \\
\hline Pleural effusion & $33(56.9)$ & 7 (63.6) & $16(59.3)$ & $10(50)$ & 0.781 \\
\hline Pneumonia & $14(24.1)$ & $1(9.1)$ & $3(11.1)$ & $10(50)$ & 0.005 \\
\hline Pneumothorax & $12(20.7)$ & $2(18.2)$ & $6(22.2)$ & $4(20)$ & 1.000 \\
\hline Pleural empyema & $9(15.5)$ & $0(0)$ & $3(11.1)$ & $6(30)$ & 0.060 \\
\hline Sepsis & $9(15.5)$ & $2(18.2)$ & $2(7.4)$ & $5(25)$ & 0.254 \\
\hline Hospital stay (days) & $31.5(6-243)$ & $18(8-54)$ & $36(6-89)$ & $42.5(18-243)$ & 0.017 \\
\hline ICU stay (days) & $9(1-200)$ & $4.5(1-12)$ & $9(1-59)$ & $21(4-200)$ & 0.003 \\
\hline Hospital readmission & $13(22.4)$ & $1(9.1)$ & $8(29.6)$ & $4(20)$ & 0.424 \\
\hline In-hospital mortality & $7(12.1)$ & $1(9.1)$ & $4(14.8)$ & $2(10)$ & 1.000 \\
\hline 90-day mortality & $8(13.8)$ & $2(18.2)$ & $4(14.8)$ & $2(10)$ & 0.789 \\
\hline
\end{tabular}

Categorical variables are expressed as number (percentage), continuous variables are expressed as median (range) 


\section{Health-related quality of life}

At the onset of the study, 25 of $58(43.1 \%)$ patients were already deceased. Of the remaining 33 patients, 20 were available for the survey. HRQoL was measured after a median follow-up of 49 months. Eight patients had been treated endoscopically, 6 had underwent surgery, and another 6 had been treated conservatively. Due to the small number of cases, we were not able to find statistically significant results, except for a single symptom scale in the EORTC QLQ-C30.

The study found a general trend of lower HRQoL in patients with non-neoplastic esophageal perforation compared to published reference data [10]. The endoscopic treatment group showed the highest GIQLI overall score and highest EORTC general health status, followed by the conservative and the surgical group, although the order varied between subscales. Surgical patients generally showed the lowest HRQoL scores.

Mean overall score for HRQoL index for GERD was 4.60 (SD 4.3). The surgical treatment group had the highest overall score with 6.2 (SD 4), indicating severe symptoms, followed by the endoscopic (4.4, SD 4.5) and the conservative group (3.3, SD 4.6). Sixty percent $(n=12)$ of follow-up patients were generally satisfied with their current health status. Only three patients showed no symptoms. The other patients reported mainly a feeling of fullness $(60 \%, n=12)$ and heartburn (50\%, $\mathrm{n}=10)$ (Fig. 2).

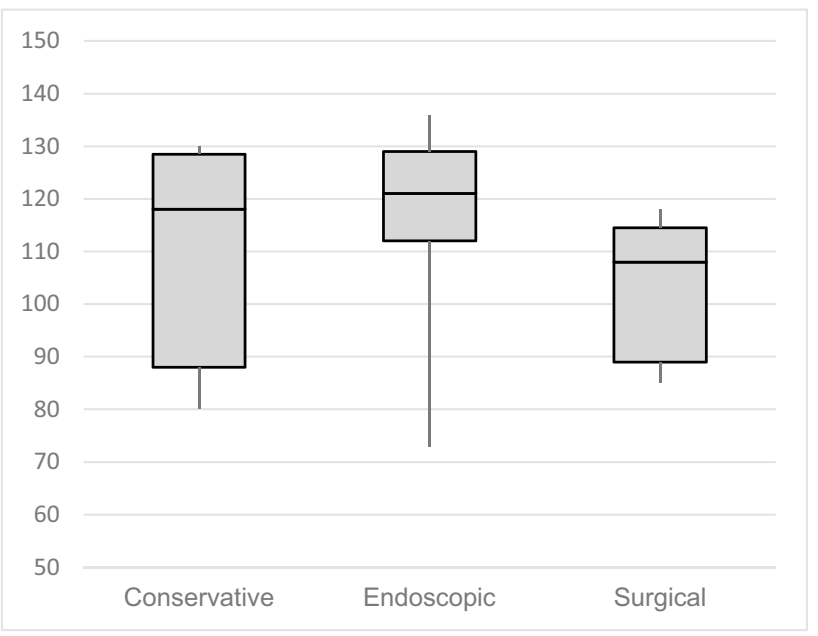

Fig. 3 Boxplots for overall GIQLI scores stratified by treatment groups (conservative $n=6$, endoscopic $n=8$, surgical $n=6$ ). Higher scores indicate better quality of life

Results of GIQLI showed a mean overall score of 111 (SD 18.9) compared to a reference score for healthy individuals of 125.8 (7). Most problems occurred in the physical function domain: $75 \%(n=15)$ of patients reported tiredness and 65\% $(n=13)$ woke up 3-4 nights of the week. All subdomains showed highest scores for the endoscopic treatment group and lowest scores for the surgical group (Fig. 3).

Global health status (GHS) in the EORTC QLQ-C30 was lower for patients with non-neoplastic esophageal
Fig. 2 Relative frequencies of symptoms in follow-up patients assessed by the HRQL for GERD. Scores $>0$ were classified as symptoms

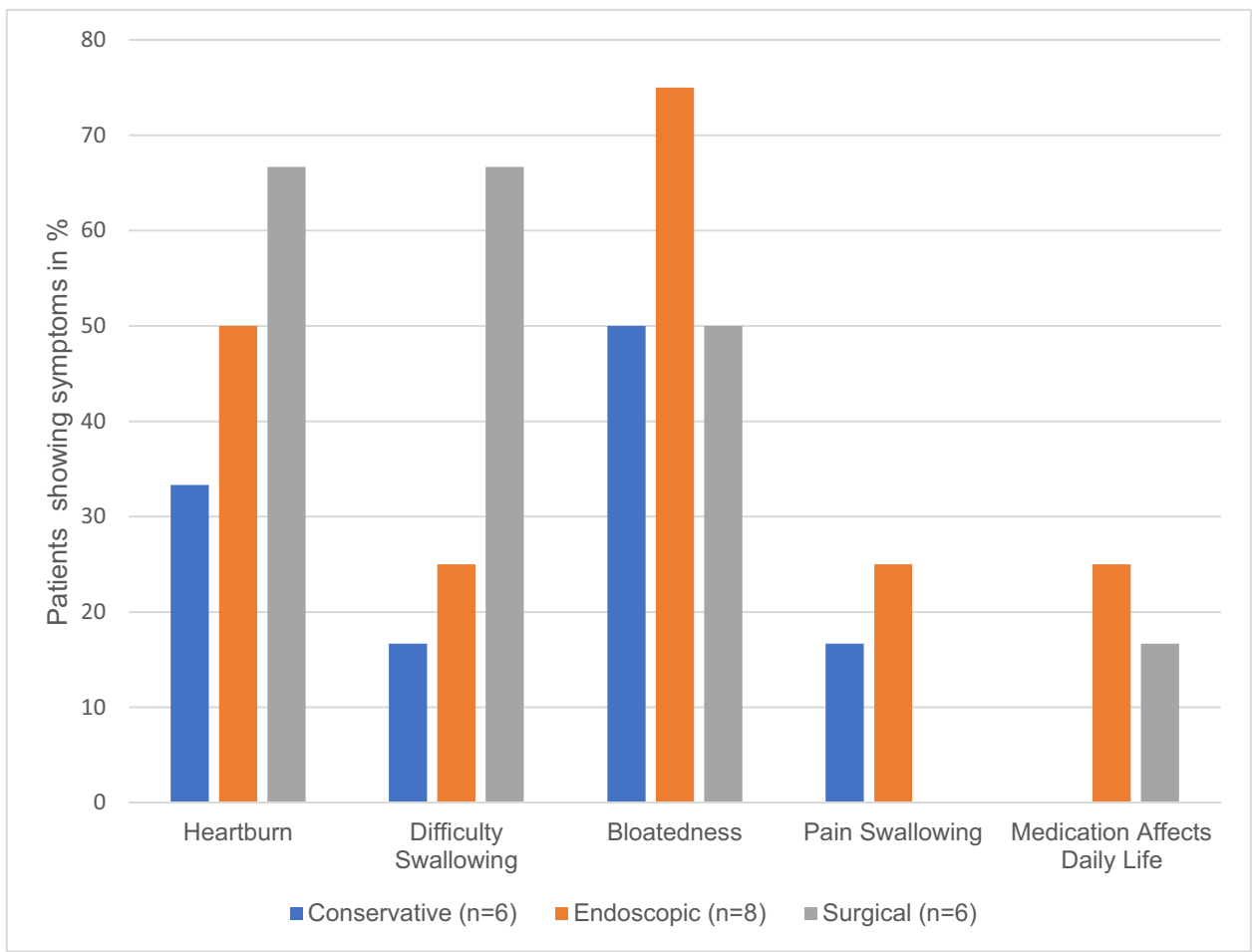


perforation than healthy individuals. Follow-up patients reached a mean score of 58.8 (SD 25.4) compared to 71.2 (SD 22.4) in the reference population [10]. Only two scores showed a statistical difference between treatment groups: Insomnia was reported by all conservatively treated patients $(100 \%, n=6)$ vs. $25 \%$ of endoscopic patients $(n=2)$ and constipation was reported exclusively by conservatively treated patients. Common symptoms were fatigue $(84.2 \%$, $\mathrm{n}=16)$ and insomnia $(60 \%, \mathrm{n}=12)$, matching the results of the GIQLI. The QLQ-C30 and QLQ-OES18 found lower function scores and higher symptom scores for the surgical group compared to conservatively or endoscopically treated patients. The GHS and functional scores are shown in Fig. 4.

\section{Discussion}

In the immediate period after esophageal perforation, especially in malignant disease, morbidity and mortality are most essential. Yet, in benign, non-neoplastic disease with normal life expectancy after successful treatment, long-term quality of life becomes the most substantial focus. Therefore, the present study was conducted to report long-term HRQOL after successful therapy of non-neoplastic esophageal perforation. In addition, the study evaluated differences between treatment modalities regarding HRQOL in the short- and long-term follow-up.

The morbidity and mortality rate in our study is consistent with previously published studies of non-neoplastic esophageal perforation. In recently published meta-analyses and studies, mortality ranges from 12 to $28 \%[1,11,12]$. Our mortality rate of $13.8 \%$ is at the lower end of published data. The median length of hospital stay was 31.5 in our patient group and 32.9 days reported by Biancari et al. [12].
The complication rate in our series is substantially higher than previously published data $[13,14]$. Yet, we were able to demonstrate a lower rate of severe complications such as sepsis (15.5\%). The most common complications in our series were pulmonary complications such as pleural effusion and pneumonia. This is consistent with large prospective studies on complications after esophagectomy $[15,16]$.

Notably and in contrast to previously published data, endoscopic treatment did not turn out to be superior to other treatment modalities in regard to short-term morbidity and mortality. Endoscopic treatment in fact led to the highest mortality in our series which in our hypothesis could possibly be caused by our low overall mortality of $13.8 \%$ [12-14, 17-19].

\section{Health-related quality of life}

We were able to show that HRQoL was generally lower in patients with non-neoplastic esophageal perforation compared to a healthy reference population. However, the reported symptoms causing the lower HRQoL were not primarily related to esophageal dysfunction, raising the question whether the used questionnaires were able to reflect HRQoL associated with esophageal function properly. Comparing HRQoL results between the different treatment groups, the study shows a trend in favor of non-operative treatment. Endoscopic treatment seems to result in better HRQoL than conservative or surgical therapy. These findings were consistent for the GIQLI and the EORTC questionnaires. Due to the small patient cohort, these results are not statistically significant. Comparing long-term follow-up, a clear confounding factor is treatment selection based on underlying complications and comorbidities. Old, multimorbid patients for example might more often be treated
Fig. 4 Global Health Score (GHS) and functional scales of the EORTC QLQ-C30 and QLQ-OES18 stratified by treatment groups (conservative $n=6$, endoscopic $n=8$, surgical $n=6)$. Higher scores indicate higher quality of life or higher level of function

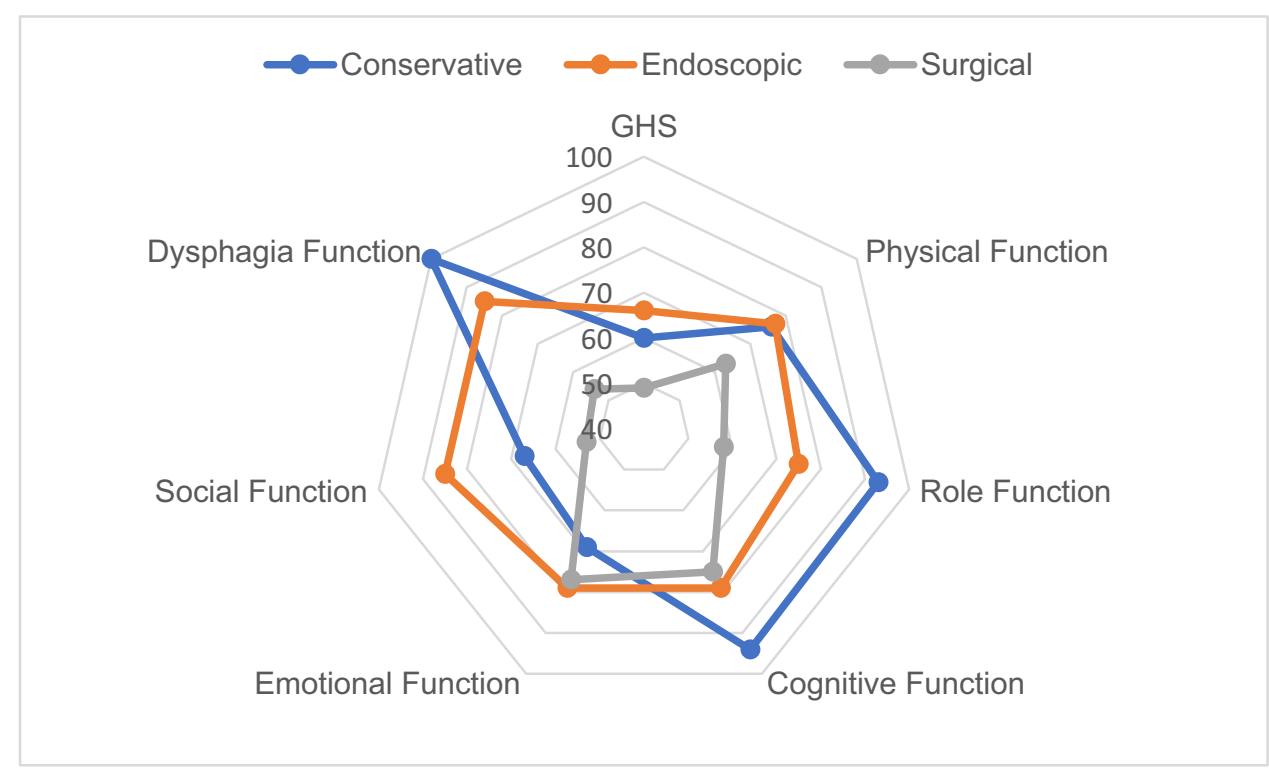


conservatively, while severe mediastinitis might lead to immediate surgery.

Several studies investigate HRQoL after esophageal surgery for malignancy. We have previously reported HRQoL after esophagectomy and transhiatal gastrectomy in patients with carcinoma of the gastroesophageal junction and were able to show that patients after esophagectomy exhibited a decreased HRQoL compared to patients after gastrectomy [20]. Yet compared to our data, EORTC questionnaires of patients after esophagectomy for cancer demonstrated a better HRQoL than surgically treated patients for perforation. Likewise, the mean Global Health Score reported by Fuchs et al. was 60.0 compared to 48.6 in our data. This superiority was consistent across subscales for physical function, role function, and emotional and social function. Furthermore, fatigue, nausea, pain, dyspnea, restlessness, and financial problems were more common in our data. This result is surprising considering the malignant nature of the underlying disease in the study by Fuchs et al.

Young et al. reported HRQoL in 81 patients with esophagectomy for benign disease such as motility disorders, esophageal strictures, hiatal hernia, and 6 patients with perforation of the esophagus using the SF-36 questionnaire [21]. With a median follow-up of 9.8 years, patients after esophagectomy showed an inferior general health status as well as physical and social function compared to general population. Keeping in mind the divergent questionnaires, this finding matches our data for surgically treated patients.

Dhayat et al. compared HRQoL of patients after endoscopic vacuum treatment for esophageal perforation or anastomotic leakage after esophagectomy and gastrectomy with patients without leakage [22]. HRQoL was evaluated with the GIQLI questionnaire with a median follow-up of 20 months. They reported a mean GIQLI score of 83 points compared to 116 points in the endoscopically treated group in our study. The superior result in our group is likely due to a higher proportion of patients with malignant disease and postoperative complications in the study by Dhayat et al.

A thorough literature review revealed three publications that exclusively focused on HRQoL after non-neoplastic esophageal perforations. Varghese et al. published a case report of four cases with reduced HRQoL after esophageal resection due to Boerhaave syndrome [23]. They only used a basic 1-10 score to evaluate HRQoL without validated questionnaires. A Norwegian group published two papers on HRQoL after esophageal perforation. One study covers bolus impactation $(n=10)$, while the other deals with iatrogenic perforations $(n=5)$ [24, 25].

All three publications are limited by small sample size. Furthermore, patient selection (surgically treated patients only, iatrogenic perforation, bolus impactation) is a confounding factor. Our study is the first to include endoscopically placed vacuum therapy in the endoscopic treatment regimen and compares outcome between different treatment modalities. In summary, in this study, we present the largest patient cohort with a thorough evaluation with the use of validated questionnaires and a direct comparison of HRQoL in different treatment modalities.

\section{Strengths and limitations}

Even though the present study is the largest observational study regarding diagnosis and number of patients, a limitation is the low number of cases within different treatment groups. This makes extensive statistical analyses difficult as well as drawing evidence-based recommendation for general clinical management.

Additional limitation is specific to the utilized questionnaires. So far, no standardized, validated, and especially designed questionnaire for patients treated for non-neoplastic esophageal perforation is available. Therefore, we used the EORTC and the GIQLI score which are commonly used to assess quality of life in upper GI patients [26]. We found that the results for the EORTC and the GIQLI score were comparable. Yet, the symptoms that lead to the diminished HRQoL were mainly not related to the esophagus. Therefore, signs of esophageal dysfunction could be underestimated in our cohort. We do believe that the use of symptom scores such as the EORTC and QLQ-C30 are advantageous, as it allows the comparison of HRQOL data to a large reference population.

Another point that must be discussed and limits the relevance of this study is its retrospective character. Due to the emergency treatment situation, we were not able to compare our data to pretreatment baseline HRQOL. A significant part of primarily successfully treated patients was already deceased at the time of questioning, and information on patients' cause of death were not available. Yet, considering the fact that the discussed disease always calls for an emergency treatment, prospective studies are unavailable and can hardly be expected in the future.

A strength of this study was the very strict inclusion criteria resulting in a homogenous patient cohort with benign, non-neoplastic disease only. Thus, results are not confounded by quality of life changes through carcinoma. With objective and validated questionnaires, data is compared to the normal population. Treatment was performed at a high-volume center for esophageal surgery with a vast experience of treating esophageal perforation and anastomotic leakage. This allowed immediate and optimal treatment based on individual patient needs and not on availability of caregivers. 


\section{Conclusion}

In this study, our historical collective was analyzed and described. In our experience, esophageal perforation ought to be treated fast and aggressively in order to minimize morbidity and mortality. Quality of life in patients with non-neoplastic esophageal perforation is still reduced in the long-term follow-up, but reported symptoms are not imperatively caused by the event of esophageal injury. New emerging endoscopic treatments proved to be a good alternative to the well-established surgical options [19]. There seems to be a trend towards endoscopic treatment. Since more endoscopic treatment was performed in the later phase of the collective, no clear recommendation for treatment can be made. We would like to emphasize that endoscopic treatment should always be seen as an option. Since the endoscopic treatment rarely seems to fail saving patients from esophagectomy, it should always be considered a valid primary treatment option, especially when one considers that short-term complication rates and the length of ICU stays as well as hospital stays in general were considerably higher after operative treatment.

Authors' contributions SB, LK, and JL were major contributors in writing the manuscript. KK was responsible for statistical analysis, and DPD was responsible for statistical analysis and radiological diagnostic of the study cohort. HF, PP, and SHC performed patient's endoscopy/ endoscopic treatment. $\mathrm{AH}, \mathrm{CB}$, and WS were responsible for surgical management of patients and helped finalizing the manuscript. All authors read and approved the final manuscript.

Funding Open Access funding enabled and organized by Projekt DEAL.

Data availability The datasets during and/or analyzed during the current study are available from the corresponding author on reasonable request.

\section{Declarations}

Ethics approval and consent to participate The protocol of this study was approved by the Ethics Committee of the University Hospital of Cologne (reference number 16-268). The informed consent from study patients was written as patients were asked to complete questionnaires.

Consent for publication Not applicable.

Competing interests The authors declare no competing interests.

Open Access This article is licensed under a Creative Commons Attribution 4.0 International License, which permits use, sharing, adaptation, distribution and reproduction in any medium or format, as long as you give appropriate credit to the original author(s) and the source, provide a link to the Creative Commons licence, and indicate if changes were made. The images or other third party material in this article are included in the article's Creative Commons licence, unless indicated otherwise in a credit line to the material. If material is not included in the article's Creative Commons licence and your intended use is not permitted by statutory regulation or exceeds the permitted use, you will need to obtain permission directly from the copyright holder. To view a copy of this licence, visit http://creativecommons.org/licenses/by/4.0/.

\section{References}

1. Sdralis EK, Petousis S, Rashid F, Lorenzi B, Charalabopoulos A (2017) Epidemiology, diagnosis, and management of esophageal perforations: systematic review. Dis Esophagus 30:1-6

2. Leers JM, Vivaldi C, Schäfer H, Bludau M, Brabender J, Lurje G, Lurje G, Herbold T, Hölscher AH, Metzger R (2009) Endoscopic therapy for esophageal perforation or anastomotic leak with a self-expandable metallic stent. Surg Endosc 23(10):2258-2262

3. Brinster CJ, Singhal S, Lee L, Marshall MB, Kaiser LR, Kucharczuk JC (2004) Evolving options in the management of esophageal perforation. Ann Thorac Surg 77:1475-1483

4. Biancari F, Saarnio J, Mennander A, Hypén L, Salminen P, Kuttila K, Victorzon M, Böckelman C, Tarantino E, Tiffet O, Koivukangas V, Søreide JA, Viste A, Bonavina L, Vidarsdóttir H, Gudbjartsson T (2014) Outcome of patients with esophageal perforations: a multicenter study. World J Surg 38(4):902-909

5. Freeman RK, Ascioti AJ, Dake M, Mahidhara RS (2015) An assessment of the optimal time for removal of esophageal stents used in the treatment of an esophageal anastomotic leak or perforation. Ann Thorac Surg 100:422-426

6. Velanovich V (2007) The development of the GERD-HRQL symptom severity instrument. Dis Esophagus 20:130-134

7. Eypasch E, Williams JI, Wood-Dauphinee S, Ure BM, Schmulling C, Neugebauer E, Troidl H (1995) Gastrointestinal quality of life index: development, validation and application of a new instrument. Br J Surg 82(2):216-222

8. Aaronson NK, Ahmedzai S, Bergman B, Bullinger M, Cull A, Duez NJ, Filiberti A, Flechtner H, Fleishman SB, de Haes JCJM, Kaasa S, Klee MC, Osoba D, Razavi D, Rofe PB, Schraub S, Sneeuw KCA, Sullivan M, Takeda F (1993) The European Organisation for Research and Treatment of Cancer QLQ-C30: a quality-of-life instrument for use in international clinical trials in oncology. J Natl Cancer Inst 85:365-376

9. Fayers PM, Aaronson NK, Bjordal K, Groenvold M, Curran D, Bottomley A, on behalf of the EORTC Quality of Life Group (2001) The EORTC QLQ-C30 scoring manual (3rd Edition). Published by: European Organisation for Research and Treatment of Cancer, Brussels

10. Scott NW, Fayers PM, Aaronson NK, Bottomley A, de Graeff A, Groenvold M, Gundy C, Koller M, Petersen MA, Sprangers MAG (2008) EORTC QLQ-C30 reference values, Brussels

11. Brinster CJ, Singhal S, Lee L, Marshall MB, Kaiser LR, Kucharczuk JC (2004) Evolving options in the management of esophageal perforation. Ann Thorac Surg 77(4):1475-1483

12. Biancari F, D'Andrea V, Paone R, Di Marco C, Savino G, Koivukangas V, Saarnio J, Lucenteforte E (2013) Current treatment and outcome of esophageal perforations in adults: systematic review and meta-analysis of 75 studies. World J Surg 37(5):1051-1059

13. Keeling WB, Miller DL, Lam GT, Kilgo P, Miller JI, Mansour KA, Force SD (2010) Low mortality after treatment for esophageal perforation: a single-center experience. Ann Thorac Surg 90(5):1669-1673

14. Schweigert M, Sousa HS, Solymosi N, Yankulov A, Fernández MJ, Beattie R, Dubecz A, Rabl C, Law S, Tong D, Petrov D, Schäbitz A, Stadlhuber RJ, Gumpp J, Ofner D, McGuigan 
J, Costa-Maia J, Witzigmann H, Stein HJ (2016) Spotlight on esophageal perforation: a multinational study using the Pittsburgh esophageal perforation severity scoring system. J Thorac Cardiovasc Surg 151(4):1002-1009

15. Biere SS, van Berge Henegouwen MI, Maas KW, Bonavina L, Rosman C, Garcia JR, Gisbertz SS, Klinkenbijl JH, Hollmann MW, de Lange ES, Bonjer HJ, van der Peet DL, Cuesta MA (2012) Minimally invasive versus open oesophagectomy for patients with oesophageal cancer: a multicentre, open-label, randomised controlled trial. Lancet 379(9829):1887-1892

16. Mariette C, Markar SR, Dabakuyo-Yonli TS, Meunier B, Pezet D, Collet D, D'Journo XB, Brigand C, Perniceni T, Carrère N, Mabrut JY, Msika S, Peschaud F, Prudhomme M, Bonnetain F, Piessen G, Fédération de RechercheenChirurgie (FRENCH) and French Eso-Gastric Tumors (FREGAT) Working Group (2019) Hybrid minimally invasive esophagectomy for esophageal cancer. N Engl J Med 380(2):152-162

17. Søreide JA, Konradsson A, Sandvik OM, Øvrebø K, Viste A (2012) Esophageal perforation: clinical patterns and outcomes from a patient cohort of Western Norway. Dig Surg 29(6):494-502

18. Vidarsdottir H, Blondal S, Alfredsson H, Geirsson A, Gudbjartsson T (2010) Oesophageal perforations in Iceland: a whole population study on incidence, aetiology and surgical outcome. Thorac Cardiovasc Surg 58(8):476-480

19 Abbas G, Schuchert MJ, Pettiford BL, Pennathur A, Landreneau J, Landreneau J, Luketich JD, Landreneau RJ (2009) Contemporaneous management of esophageal perforation. Surgery. 146(4):74955 (discussion 755-6)

20. Fuchs H, Hölscher AH, Leers J, Bludau M, Brinkmann S, Schröder W, Alakus H, Mönig S, Gutschow CA (2016) Longterm quality of life after surgery for adenocarcinoma of the esophagogastric junction: extended gastrectomy or transthoracic esophagectomy? Gastric Cancer 19(1):312-317

21. Young MM, Deschamps C, Trastek VF, Allen MS, Miller DL, Schleck CD, Pairolero PC (2000) Esophageal reconstruction for benign disease: early morbidity, mortality, and functional results. Ann Thorac Surg 70(5):1651-1655

22. Dhayat SA, Schacht R, Mennigen R, Palmes D, Vogel T, Vowinkel T, Senninger N, Laukoetter MG (2019) Long-Term quality of life assessment after successful endoscopic vacuum therapy of defects in the upper gastrointestinal tract quality of life after EVT. J Gastrointest Surg 23(2):280-287

23. Varghese D, Patel H, Waters R, Dickson G (2000) Quality-of-life study on four patients who underwent esophageal resection and delayed reconstruction for Boerhaave's syndrome. Dis Esophagus 13(4):314-316

24. Hauge T, Kleven OC, Johnson E, Hofstad B, Johannessen HO (2018) Outcome after accidental food bolus-induced esophageal perforation. Scand J Gastroenterol 53(8):905-909

25. Hauge T, Kleven OC, Johnson E, Hofstad B, Johannessen HO (2019) Outcome after iatrogenic esophageal perforation. Scand J Gastroenterol 11:1-5

26. Straatman J, Joosten PJ, Terwee CB, Cuesta MA, Jansma EP, van der Peet DL (2016) Systematic review of patient-reported outcome measures in the surgical treatment of patients with esophageal cancer. Dis Esophagus 29(7):760-772

Publisher's note Springer Nature remains neutral with regard to jurisdictional claims in published maps and institutional affiliations. 